\title{
A new paradigm for space astrophysics mission design
}

Jonathan Arenberg, Charles Atkinson, Jim Breckinridge, Alberto Conti, Lee Feinberg, et al.

Jonathan Arenberg, Charles Atkinson, Jim Breckinridge, Alberto Conti, Lee Feinberg, Charles Lillie, Howard MacEwen, Ronald Polidan, Marc Postman, Gary Matthews, Eric Smith, "A new paradigm for space astrophysics mission design," Proc. SPIE 9143, Space Telescopes and Instrumentation 2014: Optical, Infrared, and Millimeter Wave, 914315 (2 August 2014); doi: 10.1117/12.2055509

Event: SPIE Astronomical Telescopes + Instrumentation, 2014, Montréal, Quebec, Canada 


\title{
A New Paradigm for Space Astrophysics Mission Design
}

\author{
Jonathan Arenberg ${ }^{(1)}$, Charles Atkinson ${ }^{(1)}$, Jim Breckinridge ${ }^{(2)}$, Alberto Conti ${ }^{(1)}$, Lee \\ Feinberg $^{(3)}$, Charles Lillie ${ }^{(4)}$, Howard MacEwen ${ }^{(5)}$, Ronald Polidan ${ }^{(1)}$, Marc Postman ${ }^{(6)}$, \\ Gary Matthews ${ }^{(7)}$ and Eric Smith ${ }^{(8)}$ \\ ${ }^{(1)}$ Northrop Grumman Aerospace Systems, One Space Park Drive, Redondo Beach CA 90278 \\ (2) Breckinridge Associates, LLC 985 East California Blvd., Pasadena CA 91106 \\ ${ }^{(3)}$ Goddard Space Flight Center, Greenbelt MD \\ (4)Lillie Consulting LLC, 6202 Vista del Mar, Playa del Rey, CA 90293 \\ ${ }^{(5)}$ Reviresco LLC, 4091 Loosestrife Court, Annandale VA 22003 \\ ${ }^{(6)}$ Space Telescope Science Institute, XXX San Martin Drive, Baltimore MD \\ ${ }^{(7)}$ Exelis, 400 Initiative Drive, PO Box 60488 Rochester, NY 14606-0488 \\ ${ }^{(8)}$ NASA Headquarters, Washington DC
}

\begin{abstract}
Pursuing ground breaking science in a highly cost-constrained environment presents new challenges to the development of future space astrophysics missions. Within the conventional cost models for large observatories, executing a flagship "mission after next" appears to be unstainable. To achieve our nation's science ambitions requires a new paradigm of system design, development and manufacture. This paper explores the nature of the current paradigm and proposes a series of steps to guide the entire community to a sustainable future.
\end{abstract}

Keywords: Space Mission Design, Cost Scaling, Standardization

\section{Introduction}

Recently, some thought provoking work on the nature of cost scaling relationships for space telescopes and missions has been published. [1][2][3] In addition, the science cases and baseline designs for missions following the James Webb Space Telescope's (JWST) 2018 launch have also been presented. [4][5] This paper merges these works to investigate the viability of developing these proposed missions given likely available resources. Based on this initial look, we must conclude that the current paradigm for the development of space science missions is not sustainable over the long term.

The current paradigm will be reviewed and a historical analogy with pre-industrial manufacturing established. A brief examination of the cost of computing systems is given as an example where costs can be drastically reduced. The factors that transformed computer building from the work of nations to a commodity are analyzed from applicable lessons for space astrophysics. These lessons are used to guide our thoughts in the development of a road map needed to bring about this new reality.

Space Telescopes and Instrumentation 2014: Optical, Infrared, and Millimeter Wave, edited by Jacobus M. Oschmann, Jr., Mark Clampin, Giovanni G. Fazio, Howard A. MacEwen, Proc. of SPIE Vol. 9143, 914315 • (C) 2014 SPIE CCC code: $0277-786 \mathrm{X} / 14 / \$ 18 \cdot$ doi: $10.1117 / 12.2055509$ 


\section{Methodology}

In looking ahead a generation or two we are following in the footsteps of the Unites States Air Force's Project Forecast of the 1960's. [6] In this effort, the Air Force looked ahead a generation or two, to so called $\mathrm{N}+2$ or $\mathrm{N}+3$ systems, to determine a plan to successfully realize these advanced systems. We make use of a similar methodology to use the existing cost scaling relationships and apply it to our archetypal N+2 and N+3 systems. [2][3] A projection of likely NASA resources dedicated to astrophysics and a single "flagship" mission is used to calculate the time to develop these $\mathrm{N}+2$ and $\mathrm{N}+3$ systems.

The Advanced Technology Large Aperture Telescope (ATLAST) is the $\mathrm{N}+2$ archetype adopted here and a $20 \mathrm{~m}$ UV mission is chosen for the $\mathrm{N}+3$ mission. ATLAST is defined to be a $9.2 \mathrm{~m}$ visual telescope, diffraction limited at $600 \mathrm{~nm}$. [4] The UV telescope is diffraction limited at $300 \mathrm{~nm}$, has a $20 \mathrm{~m}$ diameter primary. [5] Both missions have compelling science cases that make them likely flagship mission and therefore ideal for use in this analysis.

Table 1: Parameter Values for Archetypal Systems N, N+2 and N+3

\begin{tabular}{lccc}
\hline System Generation & $\mathrm{N}$ & $\mathrm{N}+2$ & $\mathrm{~N}+3$ \\
\hline Wavelength $[\mu \mathrm{m}]-\lambda$ & 2 & 0.6 & 0.3 \\
Diameter [m]-D & 6.5 & 9.2 & 20 \\
Temperature [K]-T & 45 & 300 & 300 \\
\hline
\end{tabular}

To estimate costs of the $\mathrm{N}+2$ and $\mathrm{N}+3$ systems, we use two results from the analysis of Stahl and coauthors. The first element of our mission cost model is the cost of the optical telescope assembly (OTA), whose cost is given parametrically as

$$
O T A=C D^{1.7} \lambda^{-0.3} T^{-0.25}
$$

where $C$ is a constant, $D$ is the diameter of the primary mirror in meters, $\lambda$ is the diffraction limited wavelength in microns and $T$ is the operating temperature in Kelvin. [2] To estimate mission cost ,we make use of a second result from Stahl et al, [3] which is the parametric model of the OTA's fraction of total mission cost, $f$, which is given

$$
f=0.11+0.09 \ln (D)
$$

The total mission cost, MC, is (1) divided by (2), namely

$$
M C=\frac{C D^{1.7} \lambda^{-0.3} T^{-0.25}}{0.11+0.09 \ln (D)} .
$$


The cost estimates for the $\mathrm{N}+2$ and $\mathrm{N}+3$ missions are normalized to the $\mathrm{N}$ mission, James Webb Telescope cost, J, using (3) and the parameters from Table 1. The estimated cost for the N+2 system is 1.44J, shown as the downward solid filled triangle in Figure 1. The estimated MC for the $\mathrm{N}+3$ system is $5.45 \mathrm{~J}$, and is shown as the open hexagon in Figure 1. Figure 1 illustrates the variation of total cost (MC) due to the value of the exponent of the telescope diameter (D).

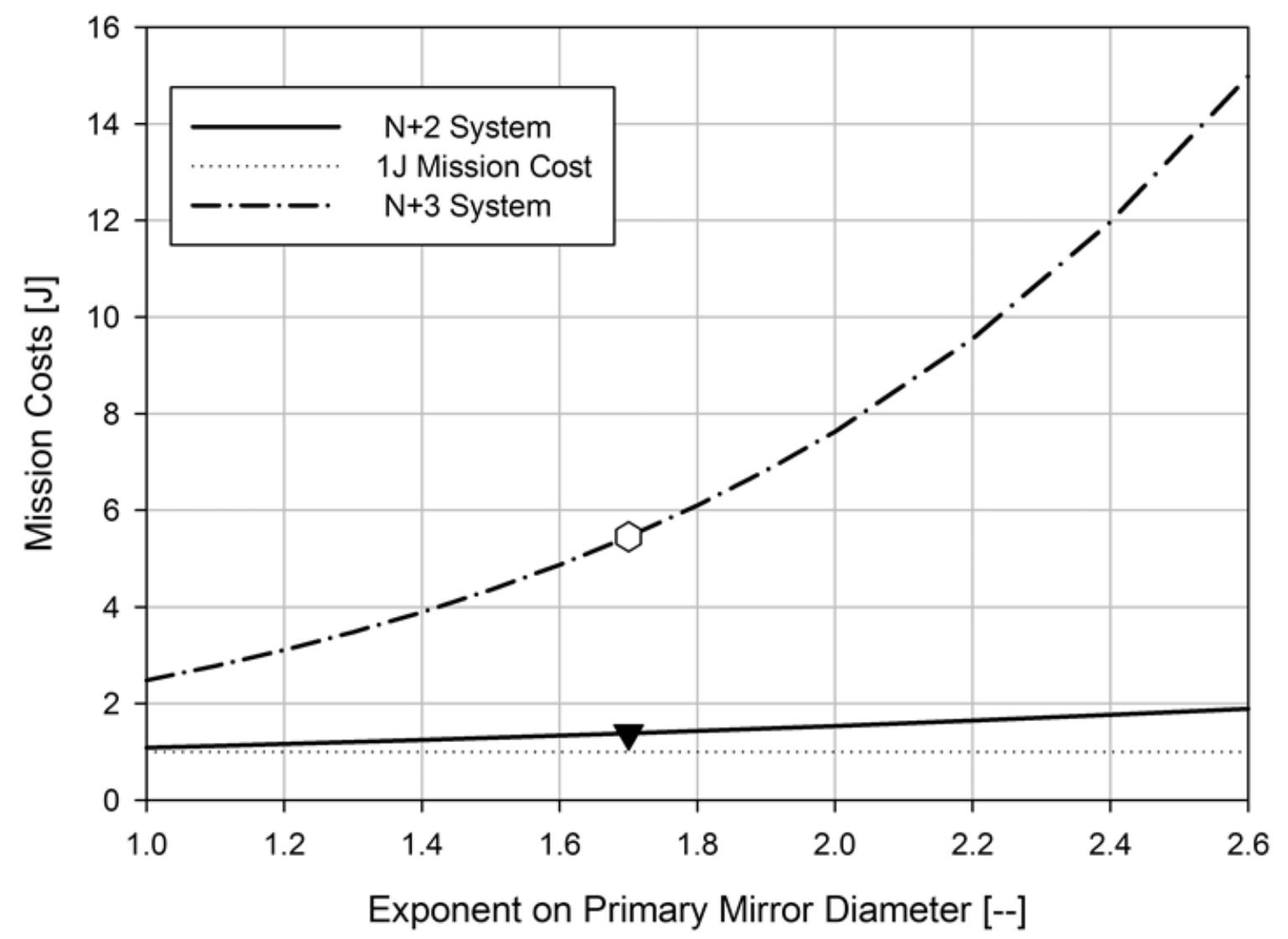

Figure 1: Mission Cost for the $\mathrm{N}+2$ and $\mathrm{N}=3$ Systems in J Units, varying the exponent on mirror diameter

Figure 2 shows the total NASA budget in 2007 dollars from 2000 through the present. [7] With the exception of the years 2008-2011, the budget is basically flat in real terms. Out of this budget approximately $7.4 \%$ has been allocated to astrophysics over the last few years. [8] For the purposes of this argument, this ratio is an expression of institutional (National) statement of the standing of space astrophysics relative to the remainder of the NASA portfolio. Of the approximately $7.4 \%$ of the NASA budget allocated to space astrophysics, the $\mathrm{N}$ generation system, JWST receives approximately $50 \%$ of this allocation. The current level of spending, 3.7\%, is our expectation for the amount of the NASA budget that will be committed to a given flagship mission in the future. 


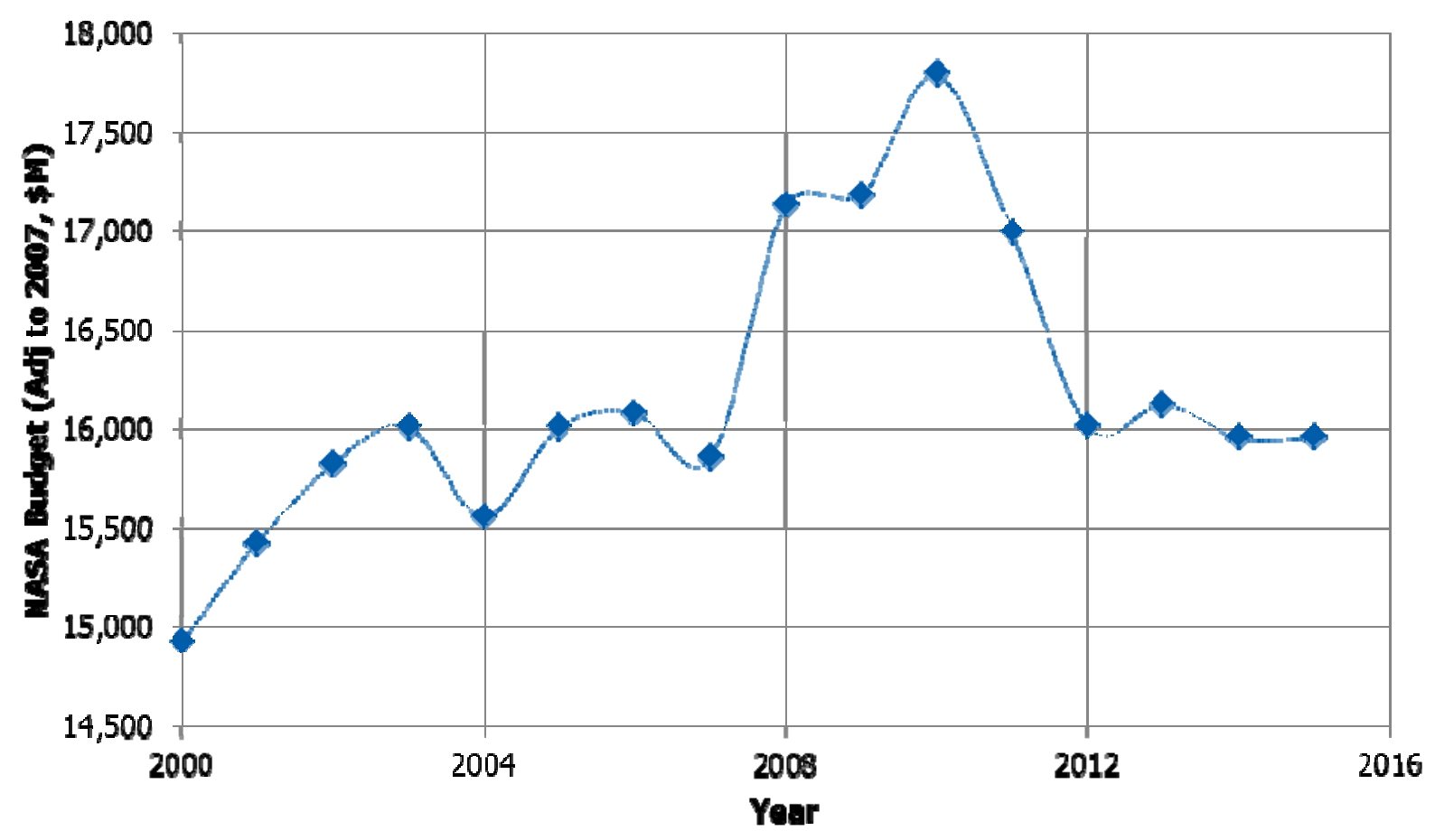

Figure 2: NASA Budget 200-2014 in 2007 Dollars

We now have a means of estimating the time for a given program to proceed from award to flight. JWST will launch approximately 16 years from contract award. Using the $\mathrm{N}$ mission as a funding yardstick, the $\mathrm{N}+2$ mission will take 23 years after award to launch and the $\mathrm{N}+3$ system will take a staggering 87.2 years to complete.

There are two factors that affect the mission developments times calculated above, the uncertainty in $M C$ and the validity of our funding model. Figure 1 shows the sensitivity of $M C$ to the value of the exponent on the primary mirror diameter. The second factor that affects the estimated development time is the funding level committed to a single astrophysics mission. Review of historical data indicates that assuming the funding level for the $\mathrm{N}$ mission will always be applied to a single mission is optimistic. Figure 3 shows budget data from 1980-2014, and shows the fraction of the NASA budget assigned to astrophysics missions, and from 1997 onward, the fraction used by JWST. If the average values over this larger history are used, the mean fraction of the NASA budget allocated to astrophysics is $6 \%$ and JWST fraction is closer to $35 \%$ of the program so far. If longer term historical norms are used as the funding level, the estimated development times increase.

Given the uncertainties in the development times for the $\mathrm{N}+2$ and $\mathrm{N}+3$ systems, time spans in decades and not years. These results make the point, that while our enterprise is sustainable through the 
current generation $\mathrm{N}$, and likely the next, it will almost certainly become unviable in the foreseeable future, possibly as early as the $\mathrm{N}+2$ mission. A very optimistic assessment, including a reduction the exponents in (3), and increased funding of the order a factor of a few, merely postpones when the paradigm breaks from the $\mathrm{N}+2$ mission to the $\mathrm{N}+3$ mission.

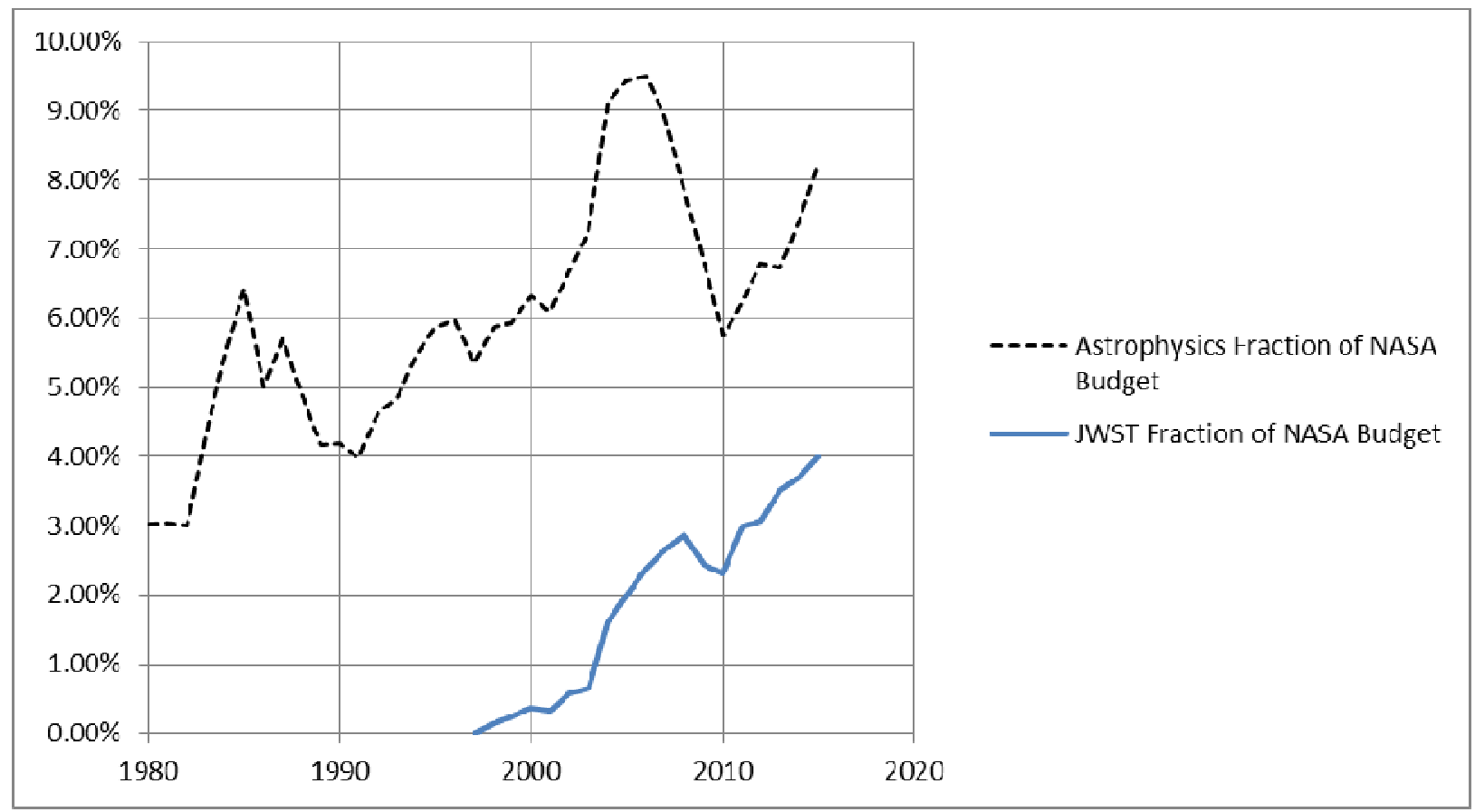

Figure 3: Fraction of NASA Budget Allocated to Astrophysics and JWST

\section{Current Space Mission Development Paradigm: Historical Analogy}

When a space science mission is chosen, it has a unique set of requirements for the hardware and on its performance in order to achieve the desired scientific return. Frequently, the hardware and technology used to meet those requirements are also unique. The supporting infrastructure in space, with the exception of launch vehicles and, in some cases, the International Space Station, is also unique.

The uniqueness of these types of large space science missions demands an artisanal approach to the design problem. This means that a large fraction of the mission cost is from unique engineering and development costs. Very little is reused from earlier missions, and when there is reuse, it is typically small cost items, propulsion tanks, transponders, communications equipment etc.

As examples of mission unique technology, Chandra X-ray Observatory had to demonstrate that the then new mirror technology was ready for launch and JWST had 10 technologies that had to be demonstrated for mission confirmation. With current missions taking 10-15 years to complete, 
engineers can reuse lessons learned only for a mission or two, due to finite working lifetimes. Manufacturing facilities are considered to be a program cost, and, therefore, are closed down as soon as the program can tolerate, limiting reuse or manufacture of "extra" stock. Test facilities, are an area where some reuse has been seen, especially on JWST, where the Chandra XRCF was modified slightly to perform interferometric tests on mirrors and structural test articles. The JWST system test will be performed at the remodeled Chamber A at JSC, which was originally built for Apollo in the early 1960s.

The current paradigm bears a striking similarity to the milieu of manufacturing prior to the industrial revolution when unique parts produced by a given workshop. Such unique parts were not readily interchangeable and lack of common interfaces made higher levels of system integration difficult and expensive.

\section{A Desirable Paradigm: Computer Cost analogy}

Computing technology is only slightly older than spaceflight but is a technology that has realized a huge decrease in costs. At inception, computers were entirely unique devices that required specialized parts and operators and were rarer than the first generation of satellites. Today, high power computing is a commodity. A modern automobile can contain 30-100 processors. [9] At the dawn of the computing age, the 1940s, the building a computer was the work of nations. Figure 3 shows the cost of a computer as a function of time, with the reference benchmark being the required amount of the then year's computing technology to produce the 2010 performance of an iPad2.

The development milieu of the first machine on the plot, ENIAC, was very similar to that of a space science mission. A small dedicated engineering team, using components designed and manufactured for other purposes and hand crafting unique parts. Even in the early days, this high expense was not a concern as it was a common understanding that the US economy needed only a few such machines. The story that follows is a familiar one, several technology leaps, the transistor, the integrated circuit, the microprocessor making it possible in the 1980 s for computing to be ubiquitous in our lives. This revolution in technology developed a huge market and competition. 


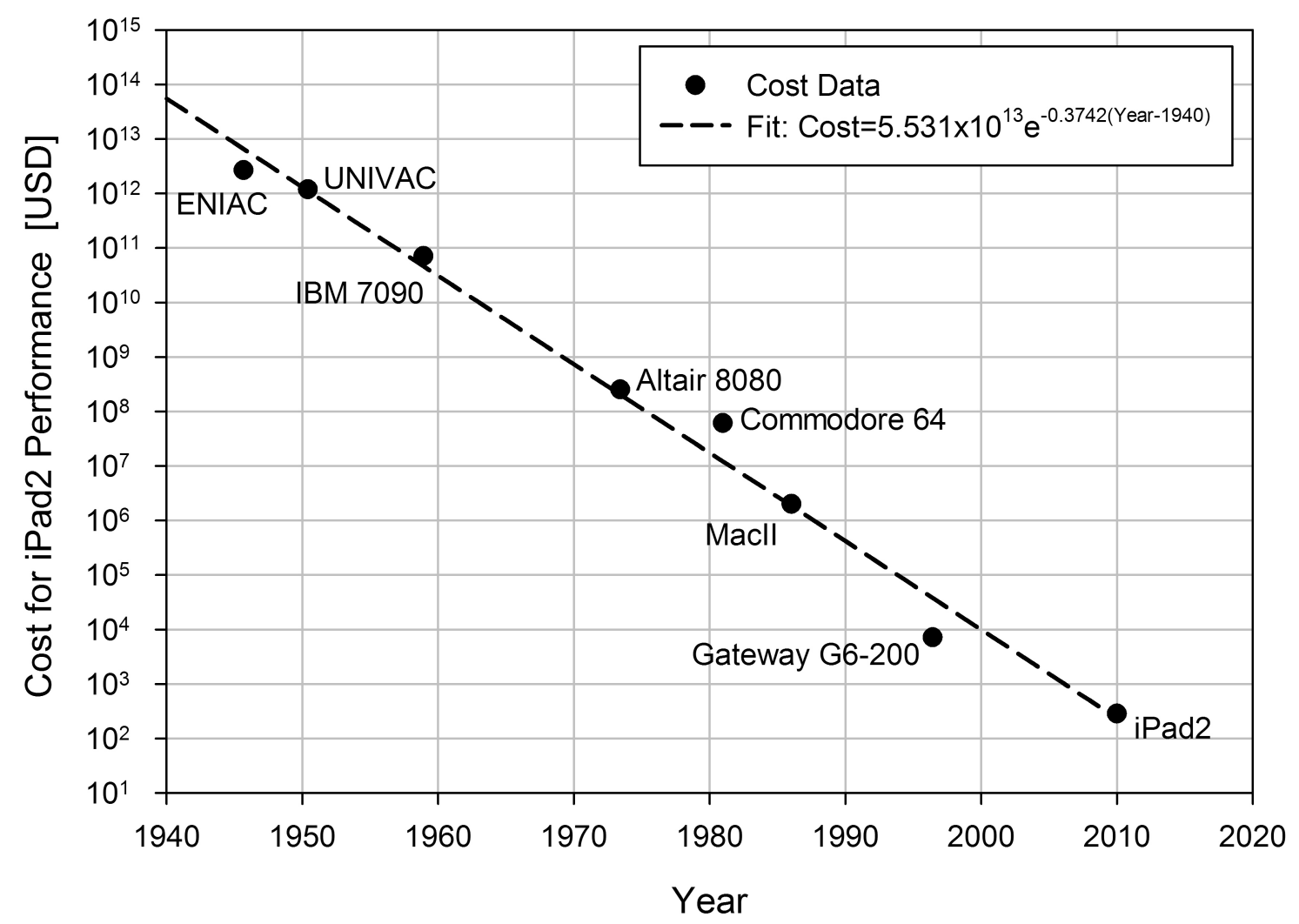

Figure 4: Cost of Computing, the estimated cost of a computer with the power of an iPad2 [10]

If the computer had not developed, an ENIAC with the computing power of a modern tablet computer would to resources approximately equal to the current US Government budget to develop!

Technology improvements in electronics are not the only reason for computing's impressive development. Some of the other factors are standardization, facilitated by industry, professional societies and standards organizations. There are extremely high levels of competitiveness among the industrial players, but also cooperation through standards working groups. The related electronics business has a super-industrial organization, SEMATECH, helping to chart the orderly development of photolithography, which is a key underlying technology. Specialization is also evident in the computer industry, hard disk manufacturers, don't make keyboards etc. The industry produces new models at a dizzying rate, so that lessons are always being plowed back into the enterprise.

\section{Roadmap to Sustainable Future}

The attributes of a sustainable future are:

1. Planned re-use of technologies, manufacturing assets, test facilities and personnel,

2. Standard interfaces, parts, analyses and testing, 
3. Reductions in cost of all aspects of the mission, not just the telescope.

Each of these attributes must be studied to see if they are realistic to achieve, are non-antagonistic and can be crafted into a coherent system.

Study 1: Determine how to make maximum re-use of technology, manufacturing and test facilities. Study 1 should identify the $\mathrm{N}+2$ and $\mathrm{N}+3$ missions that are projected to be less expensive when executed as a larger program than individually. Are there more than one chain of such missions? Can evolutionary systems span multiple generations and lower costs? [11] Could an in-space infrastructure be designed and deployed that would aid in significant cost reductions to multiple space missions, including astrophysics and other missions? Is a collaborative effort among the NASA Directorates (and possibly other organizations) to develop such an infrastructure through cost sharing a reasonable possibility?

Study 2: Where can standardized interfaces, parts and general engineering reduce cost? Can such cost reduction indeed be significant?

Study 3: The third study should look especially at the other large cost drivers in mission cost, such as instruments, testing and engineering costs. The output of these studies would be parametric cost models like those developed for the OTA. [2][3]

These three studies could be run as "sustainability project" and led from NASA Headquarters with a steering committee providing the coupling between the working groups. Ideally, the members of the study teams should be drawn from the various stakeholders in the space science enterprise including personnel from government, industry, science and professional societies (such as SPIE, AIAA, AAS).

It is worth noting that there is an active effort underway within the U.S. Government and industry to look at reduced spacecraft cost. This effort called SUMO, includes industry and NASA participation. [12]

\section{Summary}

This paper has presented estimated mission costs and development times for candidate $\mathrm{N}+2$ and $\mathrm{N}+3$ systems. Our results show that the current development paradigm is not sustainable indefinitely. WE believe that a sustainable future for space astrophysics will require reliance on a larger usage of standardized parts and technologies along with significant re-use of component level technologies and manufacturing facilities. We have defined a plan of study to determine if the ideas presented here can lead to the sustainable future for space astrophysics we all desire.

\section{REFERENCES}

[1] G.T. van Belle, A.B. Meinel and M.P. Meinel, "The Scaling Relationship Between Telescope Cost and Aperture Size for Large Telescopes," Proc. SPIE volume 5489, 563-570(2004)

[2] H.P. Stahl, T. Henrichs, A. Leudtke and M. West, "Update on Multi-variable Parametric Cost Models for Ground and Space Telescopes," Proc. SPIE 8442 (2012) 
[3] H.P. Stahl, T. Henrichs, A. Leudtke and M. West, "Update to Single-variable Parametric Cost Models for Ground and Space Telescopes," Optical Engineering, Volume 52, Number 9 (2013)

[4] M. Postman et al, "Advanced Technology Large Aperture Space Telescope: Science Drivers and Technology Developments," Optical Engineering, Volume 51, Number 1 (2012)

[5] L.D. Feinberg, J. Budinoff, H. MacEwen, G. Matthews and M. Postman, "Modular Assembled Space Telescope," Optical Engineering, Volume 52, Number 9 (2013)

[6] Project Forecast, Schriever, General Bernard A., USAF (Ret). "Technology and Aerospace Power in the 1970s," Air University Review, September-October 1969. URL:

http://www.airpower.au.af.mil/airchronicles/aureview/1969/sep-oct/schriever.html last accessed 28 May 2014

[7] http://www.whitehouse.gov/omb/budget/historicals, Table 5.2, last accessed June 1, 2014

[8] http://www.nasa.gov/pdf/750614main_NASA_FY_2014_Budget_Estimates-508.pdf, last accessed June 1, 2014

[9] http://www.nytimes.com/2010/02/05/technology/05electronics.html?_r=0 last accessed June 1, 2014

[10] Plot modified from a plot found at URL

http://www.hamiltonproject.org/multimedia/charts/cost_of_computing_power_equal_to_an_ipad2/, last accessed June 1, 2014

[11] R. Polidan, J. Breckinridge, C. Lillie, H. MacEwen, M. Flannery and D. Dailey, “An Evolvable Space Telescope for Future Astronomical Missions", Proc SPIE 9143 (2014)

[12] B.F. Collins, E.A. Nguyen and K.L. Jones, "Space Universal Modular Architecture (SUMO): Industry Consensus Interoperability Standards to Enhance Satellite Affordability and Energize the Space Industrial Base," Reinventing space Conference 2014, American Institute of Aeronautics and Astronautics, Paper AIAA-RS-2013-1104 (2013) 